\title{
ADVANCING MATERNAL AGE AT CHILDBIRTH IS ASSOCIATED WITH LESS FAVOURABLE TRABECULAR BONE MINERAL DENSITY AND TIBIAL CORTICAL BONE GEOMETRY IN YOUNG ADULT MALE OFFSPRING
}

Charlotte Verroken ${ }^{1,2}$, Hans-Georg Zmierczak², Stefan Goemaere ${ }^{2}$, Jean-Marc Kaufman ${ }^{1,2}$, Bruno Lapauw ${ }^{1,2}$ ${ }^{1}$ Department of Endocrinology and 2Unit for Osteoporosis and Metabolic Bone Diseases, Ghent University Hospital, Ghent, Belgium.

\section{Background \& Aims}

- Advancing maternal age at childbirth has been associated with a higher risk of pregnancy complications and with adverse short-term and longterm health outcomes, but little is known about the effects on offspring bone acquisition.

- We investigated associations of maternal age at childbirth with offspring bone parameters in healthy men at the age of peak bone mass.

\section{Subjects \& Methods}

- Cross-sectional, population-based sibling pair study in 689 healthy men aged 25-45 (mean 34.5 \pm 5.5 ) years.

- Offspring bone parameters include DXA-derived bone mineral content (BMC) and areal bone mineral density (aBMD) at the spine and hip, pQCTderived trabecular volumetric BMD (VBMD) at the distal radius (4\% region), and cortical vBMD and bone geometry at the radial and tibial shaft (66\% region).

- Cross-sectional associations were investigated using linear mixed-effects modeling with adjustment for offspring age, height and weight.

\section{Results}

- Mean maternal age at childbirth was $27.0 \pm 4.7$ years $(22.0 \pm 1.9$ for lowest tertile; $26.4 \pm 1.1$ for middle tertile, $31.0 \pm 3.1$ for highest tertile).

- Figure 1 shows inverse correlations of maternal age at childbirth with offspring BMC and aBMD at the lumbar spine (A) and with trabecular BMC and VBMD at the distal radius (B). No associations were found with areal bone parameters at the total hip or femoral neck.
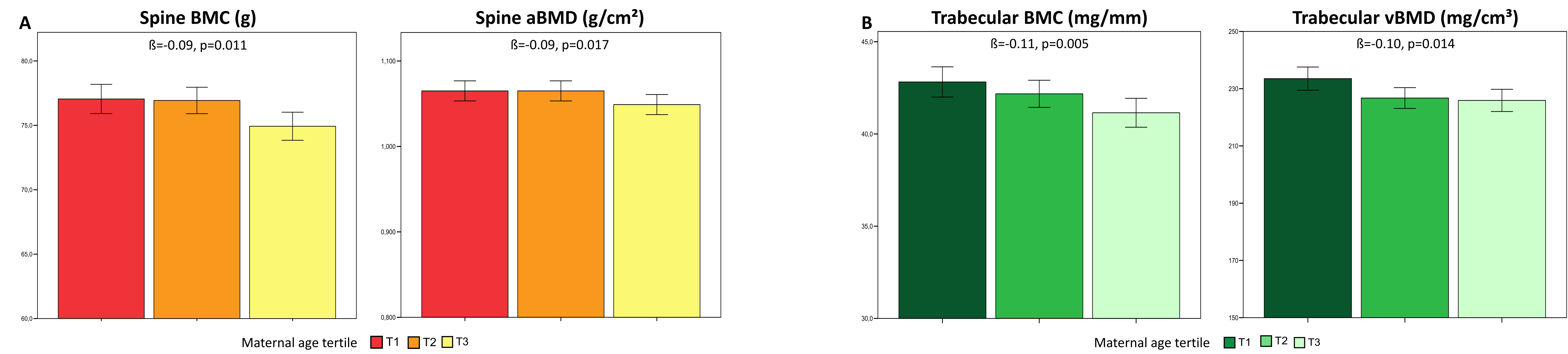

- Figure 2 shows that increasing maternal age at childbirth is associated with smaller cortical bone area, cortical over total bone area ratio, and cortical thickness at the tibial shaft in their offspring, and with larger endosteal but not periosteal circumference. No associations of maternal age were observed with cortical bone geometry at the radial shaft (data not shown) or with cortical vBMD at the radius or tibia.
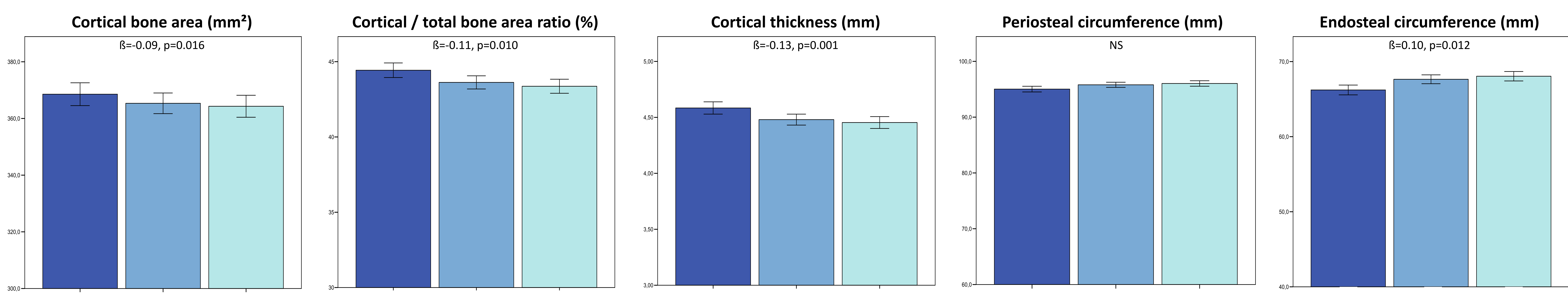

Maternal age tertile $\square \square^{\top 1} \square^{\top 2} \square{ }^{\top 3}$

Conclusions

- Advancing maternal age at childbirth might adversely affect the acquisition of peak bone mass and geometry in male offspring, with in particular less favourable trabecular bone mineral density and tibial cortical bone geometry.

- Whether this results from altered placental function or from epigenetic changes due to lifestyle factors remains to be established. 